\title{
Mental Health for Japanese People Posted Overseas
}

\author{
Chieko Kato' ${ }^{1}$, Satoshi Yoshinuma1, Koichiro Aoki', Fumiko Goto', Toshihiro Yoshizumi', \\ Takehide Goto $^{2}$, Kensei Tsuchida ${ }^{1}$ \\ ${ }^{1}$ Faculty of Information Sciences and Arts, Toyo University, Kawagoe, Japan \\ ${ }^{2}$ Faculty of Law, Toyo University, Tokyo, Japan \\ Email: milito@hotmail.co.jp
}

Received 16 January 2016; accepted 16 February 2016; published 23 February 2016

\begin{abstract}
There are an increasing number of Japanese workers living in foreign countries these days, due to globalization. The purpose of this study is to find out what kind of stress people are facing when working in China, and how they go about coping with it. Even if a person experiences stress, what is important is how he copes and deals with it in a positive way. Changing a person's cognitive thinking and how one reacts to different situations is important for people working in foreign countries. Communication, becoming involved in various activities and opening up to others will help people to cope with stress and be able to accept the culture and customs of the country in which they are living.
\end{abstract}

\section{Keywords}

Oversea Posting, Fuzzy Cluster, Stress

\section{Introduction}

There are an increasing number of Japanese workers living in foreign countries these days, due to globalization. Living in a foreign country can cause stress and affects a person's mental health. Treatment for mental health is important. People need to realize how to cope with and prevent stress. It is necessary to know how to prevent stress. According to Tsukui [1], there are four psychosocial factors which are important to find out whether people can adapt to living abroad. They include coping with stress, optimism/pessimism, motive for going abroad, and alcohol, but how people cope with stress seems to be the most important.

This study focuses on Japanese people who are posted to work in mainland China. China has its own unique culture and customs, which are different from western countries. This makes it harder for people to adapt to living and working in China. According to Furusawa [2], it has been reported to be rather stressful for some people who have been posted in China. They need to be better educated about the differences in culture and customs. This is especially true regarding mainland China, which is different from Hong Kong. The purpose of this study is to find out what kind of stress people are facing when working in China, and how they go about coping with it.

How to cite this paper: Kato, C., Yoshinuma, S., Aoki, K., Goto, F., Yoshizumi, T., Goto, T. and Tsuchida, K. (2016) Mental Health for Japanese People Posted Overseas. Open Journal of Social Sciences, 4, 30-32.

http://dx.doi.org/10.4236/jss.2016.42006 


\section{Method}

In September, 2015, 6 individuals who had been posted overseas were interviewed regarding their work and daily life. Subjects' remarks were converted to text data and classified into clusters using fuzzy cluster analysis. Subjects were grouped via fuzzy cluster analysis based on the similarity of their answers as the primary criteria. Fuzzy analysis was used to measure how frequently certain key words appear together within individual subjects' answers. Through these fuzzy techniques, ambiguity ("fuzziness”) can be defined mathematically, programmed into a computer and subsequently treated as an object to be analyzed ([3]-[5]). The definition of fuzziness is flexible, and therefore depends on the problem at hand. Fuzzy techniques are useful for mapping and comparing objects whether the degree of variation be large or small. In this study, in the grouping techniques as described in the above, subjects were grouped together based both on extreme as well as merely slight similarities in their answers.

\section{Results and Discussion}

The results of the fuzzy cluster analysis can be represented in the splitting dendrogram called "fuzzy partition tree”. Figure 1 shows the fuzzy partition tree generated from the text data of the interviewees' answers. Using fuzzy cluster analysis, the interviewees’ answers were divided into four clusters: “communication”, “stress”, “changing tasks" and “job responsibilities”.

- Communication Cluster

For workers posted overseas, the study of the language spoken in that place is key. When they are able to understand and communicate in that language-for instance, Chinese - this is good for both their study and their personal growth. When, on the other hand, they find themselves unable to learn the language sufficiently-such as in cases where their posting is too short-they can end up being isolated and this can also have a negative impact on their work. This problem is exacerbated in cases where "top-down management" practices are in place. Communication transcends nationality, is not just a matter of Japanese or Chinese, but is a matter of cooperative effort by both sides, and is best achieved when both sides make an earnest effort to understand one another. In cases where those in positions of power imagine themselves to be in a superior position, true communication from the heart becomes difficult, if not impossible.

- Stress Cluster

People in different countries have different values, and the values of the local culture should be given precedence. In places less fastidious than Japan about doing things in a certain way, this can lead to friction and stress. A manager who attempts to live entirely by Japanese values needs to adjust those values to suit those of the local culture so as to live more harmoniously with it. When this goes uncorrected, stress can lead to a lack of trust in the local workforce, and even to mental breakdowns and unreasonable or even violent behavior toward local workers. Those who are able to adjust their values to suit the local culture are better able to release their stress and work more effectively.

- Changing Tasks Cluster

Work tasks change when one is posted overseas, and while this can be challenging, it also requires a worker to meet many new people and accomplish a variety of tasks, which can be a very good experience, as such persons are free to develop strategies for coping with these new tasks freely. While such situations may be difficult, they can lead to greater flexibility and a broader perspective, both of which bring positive results. This is one of the most important and useful aspects of being posted overseas.

- Job Responsibilities Cluster

In China, it is not common for workers to share the sense of responsibility toward their work that is expected of their superiors. Most people seem to be overly detached from their work, quitting when it suits them, and showing an attitude of fatalism when things go wrong. However, there are those among the Chinese who do in fact feel a sense of responsibility toward their work, and it is important to reach out to such persons and capitalize on their willingness to assert themselves. For Japanese workers posted abroad, their new work place may represent a significant step up in position and responsibility-for instance, one Japanese worker might be assigned to work with a hundred Chinese-but this can also lead to an immense sense of pressure. Therefore, while it is generally thought to be a good thing to rise to a position of importance, it is equally important for such workers not to place undue levels of stress on themselves. 


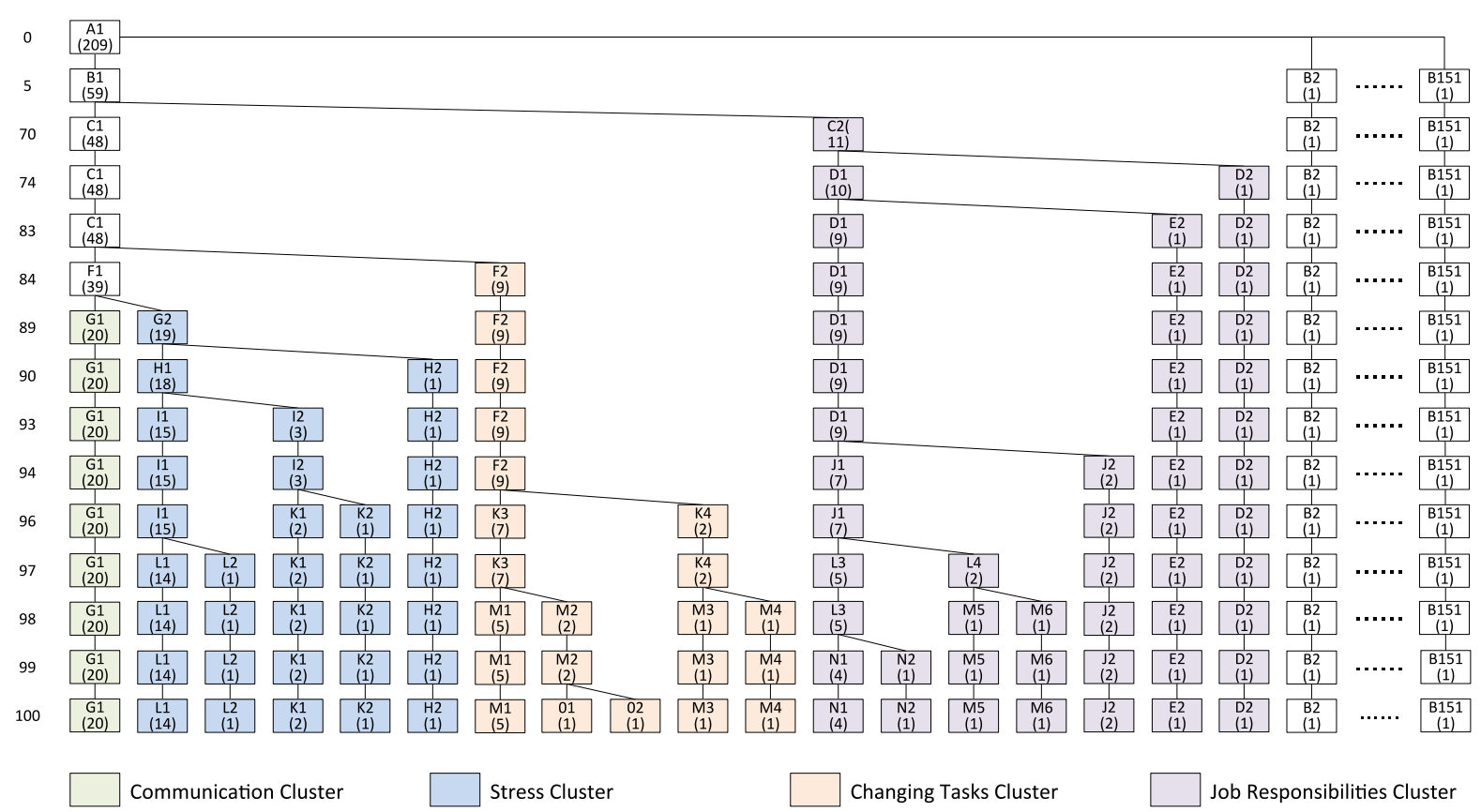

The left side value in Figure 1 represents the similarity between responses, while the rectangles placed on the right side represent formed clusters depending on the degree of similarity. Each figure within the rectangles represents the number of responses belonging to the cluster.

Figure 1. The results of fuzzy cluster analysis.

\section{Conclusion}

Even if a person experiences stress, what is important is how he copes and deals with it in a positive way. Changing a person's cognitive thinking and how one reacts to different situations is important for people working in foreign countries. Communication, becoming involved in various activities and opening up to others will help people to cope with stress and be able to accept the culture and customs of the country in which they are living.

\section{References}

[1] Tsukui, K. (1996) The Mental Health of Japanese Overseas Workers and Their Families. Japanese Journal of Psychosomatic Medicine, 36, 175-182.

[2] Furusawa, M. (2011) A Study on Human Resource Management of Japanese Expatriates: Empirical Research to Repatriates. The Review of Osaka University of Commerce, 6, 1-22.

[3] Ueseu, H. (2006) Structure Analysis of Fuzzy Node Graph and Its Application. Biomedical Soft Computing and Human Sciences, 11, 41-49.

[4] Shinkai, K. (2008) Fuzzy Cluster Analysis and Its Evaluation Method. Biomedical Soft Computing and Human Sciences, 13, 3-9.

[5] Kato, C., et al. (2014) Analysis of Fuzzy Cluster for Mental Health. IEICE Transactions on Information and Systems, 7 , 1923-1926. http://dx.doi.org/10.1587/transinf.E97.D.1923 